\title{
EchoGéo
}

19 | 2012

Du littoral à la haute mer : quelles recherches récentes en géographie?

\section{Forêt et conservation des dunes littorales : le cas exemplaire de la Réserve biologique domaniale de la Côte d'Opale (Pas-de-Calais)}

\section{Yves Petit-Berghem}

\section{OpenEdition}

Journals

Édition électronique

URL : https://journals.openedition.org/echogeo/12766

DOI : $10.4000 /$ echogeo.12766

ISSN : 1963-1197

Éditeur

Pôle de recherche pour l'organisation et la diffusion de l'information géographique (CNRS UMR 8586)

Référence électronique

Yves Petit-Berghem, «Forêt et conservation des dunes littorales : le cas exemplaire de la Réserve

biologique domaniale de la Côte d'Opale (Pas-de-Calais) », EchoGéo [En ligne], 19 | 2012, mis en ligne le 10 février 2012, consulté le 11 août 2021. URL : http://journals.openedition.org/echogeo/12766 ; DOI : https://doi.org/10.4000/echogeo.12766

Ce document a été généré automatiquement le 11 août 2021.

EchoGéo est mis à disposition selon les termes de la licence Creative Commons Attribution - Pas d'Utilisation Commerciale - Pas de Modification 4.0 International (CC BY-NC-ND) 


\title{
Forêt et conservation des dunes littorales : le cas exemplaire de la Réserve biologique domaniale de la Côte d'Opale (Pas-de-Calais)
}

\author{
Yves Petit-Berghem
}

\section{Introduction}

1 La Réserve biologique domaniale de la Côte d'Opale, créée en 1985, est un site emblématique du littoral de la Manche. S'inscrivant dans un territoire très urbanisé entre les communes de Berck-sur-Mer et de Merlimont (figures 1a et 1b), deux stations balnéaires très fréquentées, cet ensemble dunaire constitue un espace de référence, un laboratoire à ciel ouvert pour l'étude de la gestion conservatoire des milieux. Le site héberge une grande diversité d'habitats, des formes dunaires en constant remaniement, et une forêt littorale dont l'originalité dénote par son caractère subnaturel (Dereix, 2001; Paskoff, 2003). La réserve est gérée depuis des années par l'office national des forêts mais les forestiers ne travaillent pas seuls: leurs tâches multiples découlent directement des objectifs que l'ONF a fixés au site, à travers le plan de gestion et en lien étroit avec les scientifiques. Ces objectifs sont entre autres la protection d'un milieu rare et d'une qualité reconnue à l'échelle européenne ; l'étude de ce milieu, de sa biodiversité et de sa dynamique naturelle, tant géomorphologique qu'écologique; l'expérimentation de méthodes de gestion légères, peu coûteuses et respectueuses de la libre expression de la dynamique dunaire... pour aboutir à un modèle de "dune stable dans sa dynamique naturelle» (Dermaux, 2007). Pendant longtemps, la fréquentation de ce site n'a pas été encouragée (pas de sentier balisé, de panneaux d'information, de parking, etc.). Sans être exclue, puisque l'accès depuis la mer n'a jamais été interdit, son ouverture fut jusqu'à l'automne 2010 réservée aux représentants de la communauté scientifique ou aux professionnels de l'environnement. La problématique d'ouverture vers l'extérieur, en particulier vers le 
grand public, relève d'une orientation nouvelle et les forestiers doivent à la fois composer avec des acteurs inscrits dans un territoire et avec une société attirée par de nouvelles aménités. Les enjeux d'hier ne sont plus ceux d'aujourd'hui et la culture du forestier doit pouvoir s'y adapter.

Figure 1a - Situation du système dunaire de Berck-Merlimont

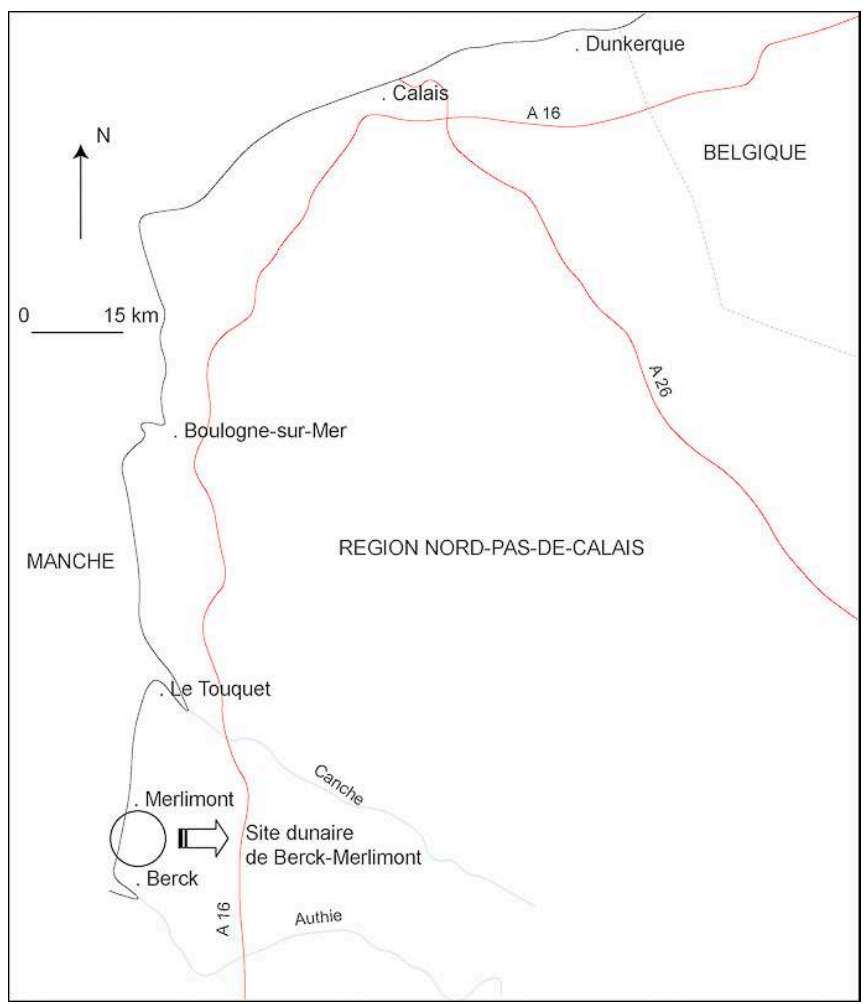


Figure 1b - Le découpage foncier du site de Berck-Merlimont

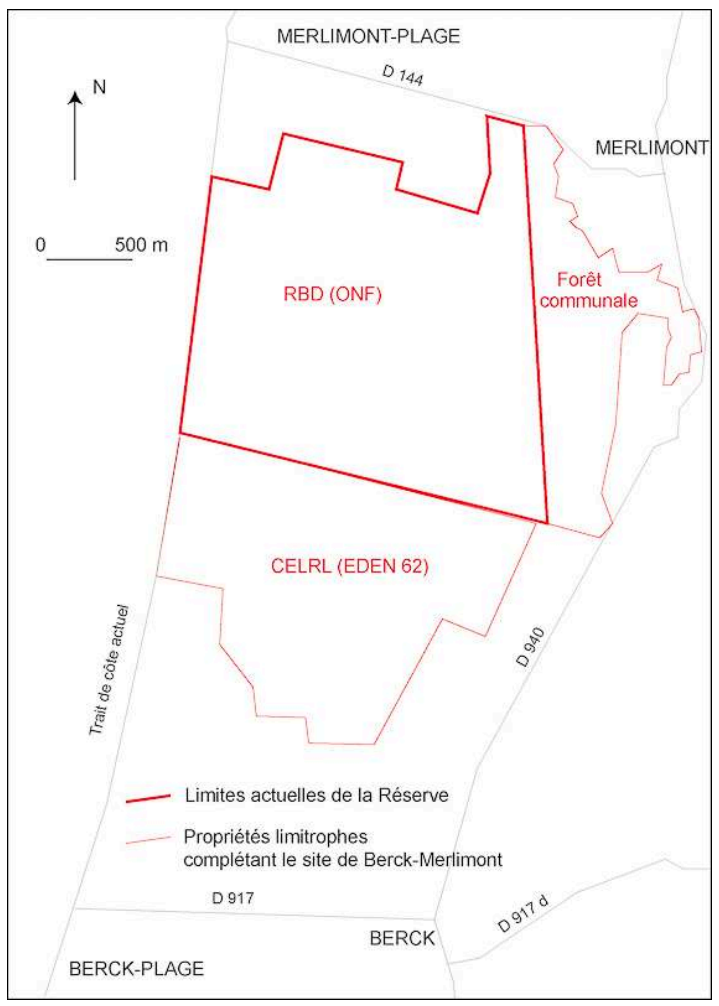

2 Par l'étendue de ses missions, l'ONF est sorti du bois depuis presque une trentaine d'années lorsqu'il fut décidé de transformer une réserve foncière scindée en plusieurs propriétés privées en réserve biologique aux mains d'un seul gestionnaire. Si cette situation s'imposa comme le résultat d'une volonté politique forte en matière de préservation du littoral, elle pose aujourd'hui le problème de la mainmise d'un organisme sur un espace entre nature et culture au sein duquel gravitent des acteurs qui n'ont pas la même perception des lieux et la même sensibilité que les forestiers. Ces acteurs ont aussi tout simplement envie d'exister sans entrer forcément dans une culture de conflits. Après avoir présenté le site et les enjeux liés à sa gestion conservatoire, des éclairages seront donnés sur le positionnement scientifique de l'ONF, sa politique en matière de conservation des écosystèmes et d'ouverture au public. Un recadrage historique sera opéré afin de contextualiser la réflexion et montrer que le temps qui s'applique ici à la grande échelle des processus phytodynamiques et aux séquences paysagères historiques, impulse un double systèmes de contraintes, naturelles et humaines, spatiales et sociales.

\section{Présentation géographique du site et des enjeux liés à sa gestion}

En bordure de la Manche, entre les stations touristiques de Berck et de Merlimont (Pasde-Calais), un important massif dunaire de plus de 800 ha, préservé de l'urbanisation, constitue un site naturel d'un intérêt exceptionnel. Il s'inscrit dans la plaine maritime picarde en bordure d'une mer dont le niveau a connu des variations de plus de $100 \mathrm{~m}$ au Quaternaire récent. Le système dunaire forme un vaste ensemble qui s'étale sur 2 à 3 $\mathrm{km}$ de large (figure 2), il comprend deux cordons dunaires (l'un externe située le plus 
proche de la mer, l'autre interne plus ancien fermant à l'arrière le système) séparés par une large plaine humide interdunaire. A l'intérieur des terres, les dunes sont séparées de la bordure d'un plateau crayeux par un ancien marais maritime aujourd'hui poldérisé situé sous le niveau des plus hautes mers.

Figure 2 - Unités écologiques de la Réserve biologique domaniale de la Côte d'Opale

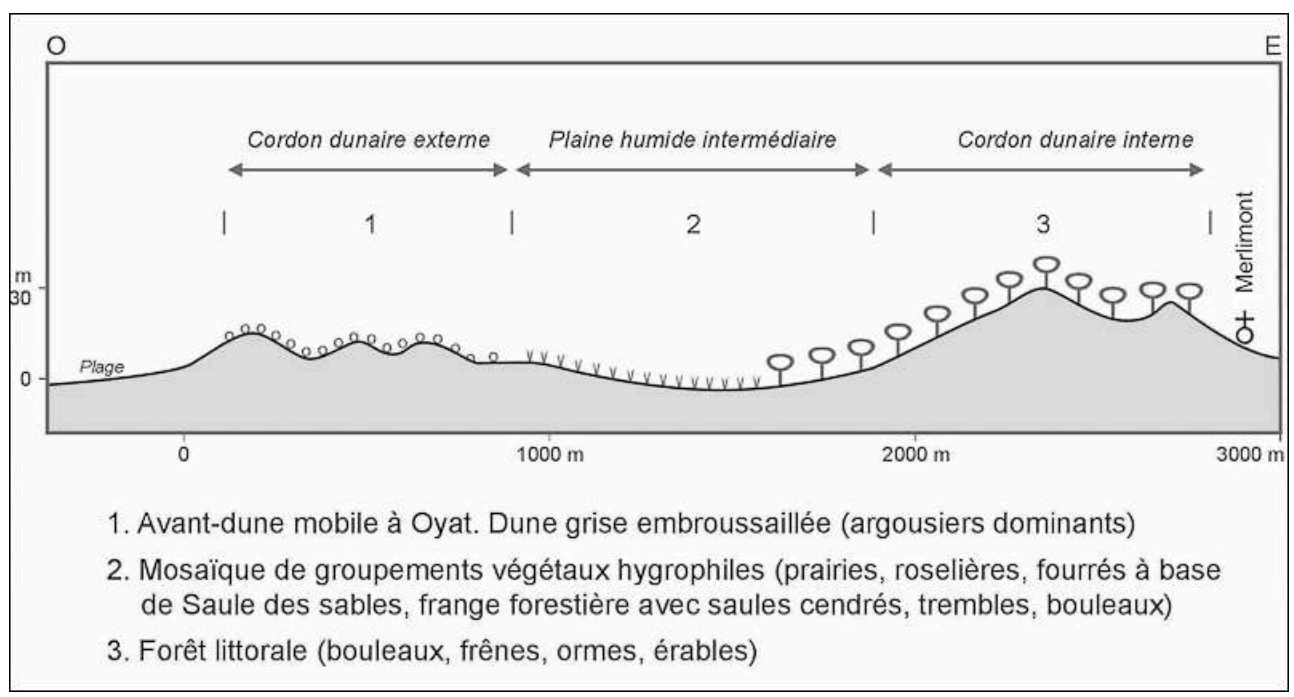

4 Sur le plan géodynamique, le site est marqué par la triple action de la mer, du vent et de la végétation. La grande largeur du système explique la diversité des morphologies rencontrées, véritable musée des formes dunaires, dont la qualité en fait sans doute l'un des plus riches en Europe du Nord-Ouest. La richesse en termes de biodiversité végétale et animale est également reconnue, le site ayant été choisi comme l'un des chantiers pilotes du projet européen Life 92 "Biodiversité et protection dunaire » centré sur la gestion des dunes littorales non boisées de la côte atlantique (Favennec et Barrère, 1997).

5 Le massif dunaire dépend aujourd'hui de trois propriétés (figure 1b). La principale relève du ministère en charge de l'Agriculture et de la Forêt, il s'agit de la dune domaniale (450 ha) dont la gestion est confiée à l'office national des forêts. Depuis 1985, cette dune est classée en Réserve biologique domaniale dirigée dite de la Côte d'Opale. Cette partie domaniale résulte de la fusion de quatre propriétés achetées entre 1972 et 1985 et marque une volonté publique forte de préservation du littoral. La seconde propriété est la dune communale de Merlimont (150 ha). Pour sa gestion, l'ONF apporte un soutien technique à la commune dans le cadre du régime forestier. La dune du Conservatoire du littoral $(290 \mathrm{ha})$ complète la trame foncière au sud du domaine et sa gestion est confiée au département (EDEN 62). Bien que multiforme, le statut foncier bénéficie à l'ONF qui est à la fois gestionnaire et force de propositions pour ses partenaires.

6 Les enjeux du site sont liés à sa biodiversité et à son caractère d'exemplarité pour la connaissance et la gestion des milieux dunaires européens (Favennec, 2007).

La biodiversité repose sur la configuration du site, deux ensembles d'habitats liés à la topographie: l'un, xérophile, caractérise les dunes sèches des cordons dunaires; l'autre, hygrophile, regroupe les habitats des pannes ${ }^{1}$ et de la vaste plaine humide interdunaire. Toutes les communautés végétales potentielles peuvent s'y exprimer, depuis les habitats pionniers sur sables minéraux des niveaux les plus bas jusqu'aux 
bas-marais tourbeux et à la forêt hygrophile naturelle dominée par le bouleau pubescent (photographie 1). C'est cette forêt littorale dite du "Ligustro-Betuletum » qui a suscité les premiers travaux de la communauté scientifique régionale et contribué fortement à la reconnaissance du système et à son classement en réserve biologique domaniale dirigée. La forêt s'accompagne d'un grand nombre de milieux associés, une soixantaine d'habitats différents dont les deux tiers relèvent de la directive européenne 92/43, directive « Habitats ». Ces complexes d'habitats constituent les supports de près de 500 espèces végétales dont 44 sont protégées au niveau régional, 7 au niveau national et une (Liparis de Loesel) répertoriée en annexe II de la directive " Habitats ». La diversité végétale se répercute sur les autres règnes du vivant avec en particulier plus de 1000 espèces de champignon.

La Réserve biologique domaniale de la Côte d'Opale constitue par ailleurs un laboratoire grandeur nature pour l'étude des dynamiques des milieux dunaires, ce qui lui confère un caractère d'excellence et d'exemplarité pour identifier et comprendre les processus qui conditionnent la diversité paysagère et écologique de ce complexe dunaire. Des études multidisciplinaires (géomorphologie, botanique, hydrogéologie, sociologie, histoire) ont déjà permis d'éclairer la compréhension des paysages et phénomènes actuels (Petit-Berghem et Dubois, 1995; Favennec et Barrère, 1997). Les apports scientifiques ont renforcé la nécessité de conserver ou recréer les processus qui sont à l'origine de la biodiversité du site, par exemple renforcer ceux liés à la dynamique active du système (érosion éolienne, fluctuations de la nappe phréatique) et freiner ceux qui sont sources de banalisation ou d'appauvrissement (expansion de plantes opportunistes comme l'argousier, anciens fossés de drainage). L'enjeu central est donc de bien comprendre le fonctionnement de ce système afin d'en tirer des enseignements pour la gestion future du site.

Photographie 1 - De la dune vive à la forêt arrière-littorale

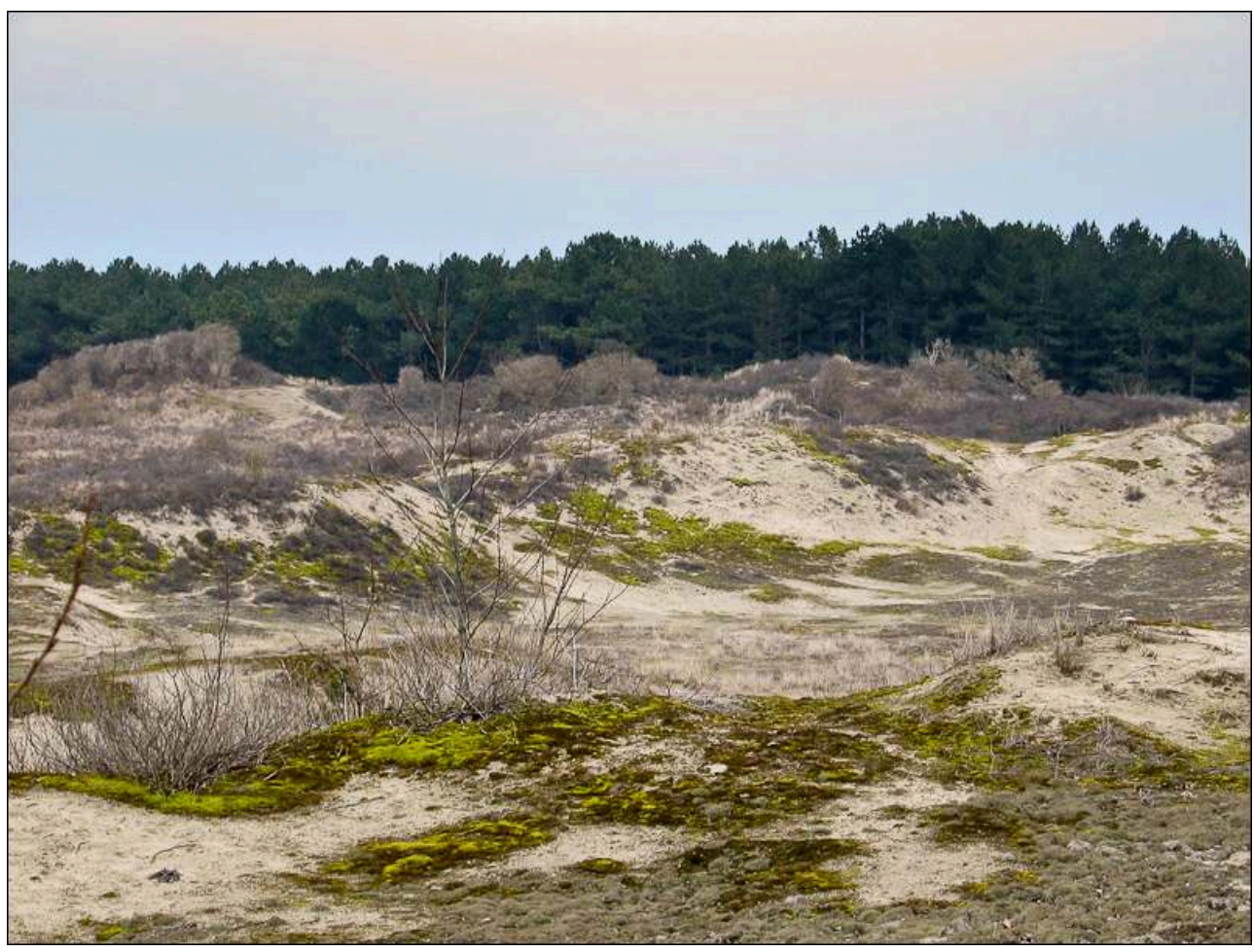

Source : cliché personnel de l'auteur, 2010. 


\section{Le statut de réserve et ce qu'il implique}

7 Le statut de réserve biologique dirigée a pour conséquence immédiate d'orienter les objectifs de gestion. Ainsi, il s'agit de protéger et assurer la gestion conservatoire d'habitats naturels particulièrement intéressants ou rares, d'espèces rares ou menacées de la faune ou de la flore, voire d'autres ressources du milieu naturel (eau douce, sable maritime, etc.). Il ne s'agit pas pour autant de sanctuariser un espace naturel, de le mettre sous cloche à l'écart du public, d'autant plus que le poids de l'histoire démontre à chaque fois le fort impact des usages humains sur l'évolution des paysages de la réserve et leur interaction permanente avec les processus naturels. Toute la difficulté du gestionnaire est donc d'orienter la gestion dans un sens conservatoire mais sans être complètement déconnecté des attentes sociales.

Ici, bien que la forêt constitue le principal écosystème, il faut parfois savoir en sortir afin de s'occuper de zones tampons pour lesquelles des règles spécifiques de gestion sont établies en fonction des objectifs propres à la réserve. L'ONF a toute la légitimité pour intervenir dans la réserve et sa politique est définie dans le cadre d'une logique de réseau national. L'ONF met en place un comité consultatif de gestion mais l'organisme garde la main pour orienter les débats et déterminer les conditions dans lesquelles les scientifiques peuvent effectuer des études et des recherches qui doivent avoir rapidement un caractère opérationnel.

Depuis la création de la réserve, l'ONF entretient des relations étroites avec quelques partenaires institutionnels dont les principaux relèvent de l'État ou des collectivités : Conservatoire du littoral, DREAL, Conseil général (Syndicat mixte EDEN 62), Conseil régional. Ces relations s'établissent dans un cadre de réflexion normatif où l'objectif est d'assurer en premier la conservation de la biodiversité biologique par l'application des plans de gestion à long terme définis dans les aménagements forestiers. La réserve est en effet une zone de référence où praticiens et chercheurs peuvent mesurer en partenariat l'incidence des pratiques de gestion et mettre au point des méthodes d'évaluation et de suivi du milieu. Mais cette démarche prônée par l'ONF reste avant tout biocentrique et n'est pas toujours comprise en particulier par tous ceux qui sont éloignés de la sphère cognitive des structures gestionnaires. Des difficultés sur lesquelles nous reviendrons sont significatives des obstacles auxquels se heurte l'aboutissement de cette démarche : les collectivités locales y voient par exemple moins une gestion cohérente de l'espace qu'une entrave de plus dans la mise en valeur de leur territoire, l'accent étant mis sur la protection de l'environnement. Celle-ci ne doit pas en effet s'opposer systématiquement aux exigences du développement local : politique foncière d'urbanisation du littoral, accueil et cheminement des touristes de la plage à l'arrière dune, politique de défense du littoral et de gestion du trait de côte, valorisation de la filière-bois, etc. Par ailleurs, il est clair que le statut de protection limite plus strictement le degré d'ouverture au public : l'accueil du public n'y est pas privilégié mais reste possible dans le respect des équilibres biologiques. En dehors des ateliers scientifiques, des chantiers nature, ou des visites pédagogiques, quelle offre pour l'usager, le visiteur de passage, ou le touriste attiré par la beauté et l'étrangeté de cet espace de nature? L'ONF reconnaît depuis peu la nécessité de s'ouvrir plus amplement vers l'extérieur : "Sans perdre de vue les objectifs de gestion retenus pour la réserve biologique domaniale, il apparaît nécessaire aujourd'hui d'envisager une plus 
large concertation autour de la gestion du site en associant les élus locaux aux décisions pour passer du concept de "partage de l'espace", avec d'un côté des dunes ultrafréquentées et de l'autre des dunes ultra-protégées, au concept « d'espace partagé » où la fréquentation du site par le public ne compromettrait en rien le maintien de la biodiversité ni ne contrarierait les expérimentations menées " (Dermaux, 2007, p. 42). Cette volonté d'ouverture appelle deux remarques : elle est tout d'abord très récente (photographie 2) alors que des pressions de différente nature (élus locaux, fréquentation touristique, chasse) existaient dès la création de la réserve. Le développement touristique naît même au début des années 1970 faisant des dunes de Berck-Merlimont l'une des "Fenêtres vertes sur la Côte d'Opale». La seconde observation traduit pour l'ONF un besoin de communiquer autrement: gagner en pragmatisme et en efficacité en élargissant son cadre de réflexion et d'intervention, aller au-delà de simples collaborations techniques et de limites administratives, faire reconnaître la pluralité des tâches de ses agents dans le contexte difficile de la restructuration de l'établissement. Ainsi, un document de gestion unique pour l'ensemble du massif est apparu nécessaire afin de dégager des synergies, de renforcer des collaborations qui jusqu'alors n'étaient qu'informelles ou balbutiantes. L'histoire du lieu peut apporter quelques éclairages pour mieux comprendre l'attitude de l'ONF et ses prises de positions dans la gestion de cette réserve.

Photographie 2 - La Réserve biologique domaniale de la Côte d'Opale : un accès réglementé

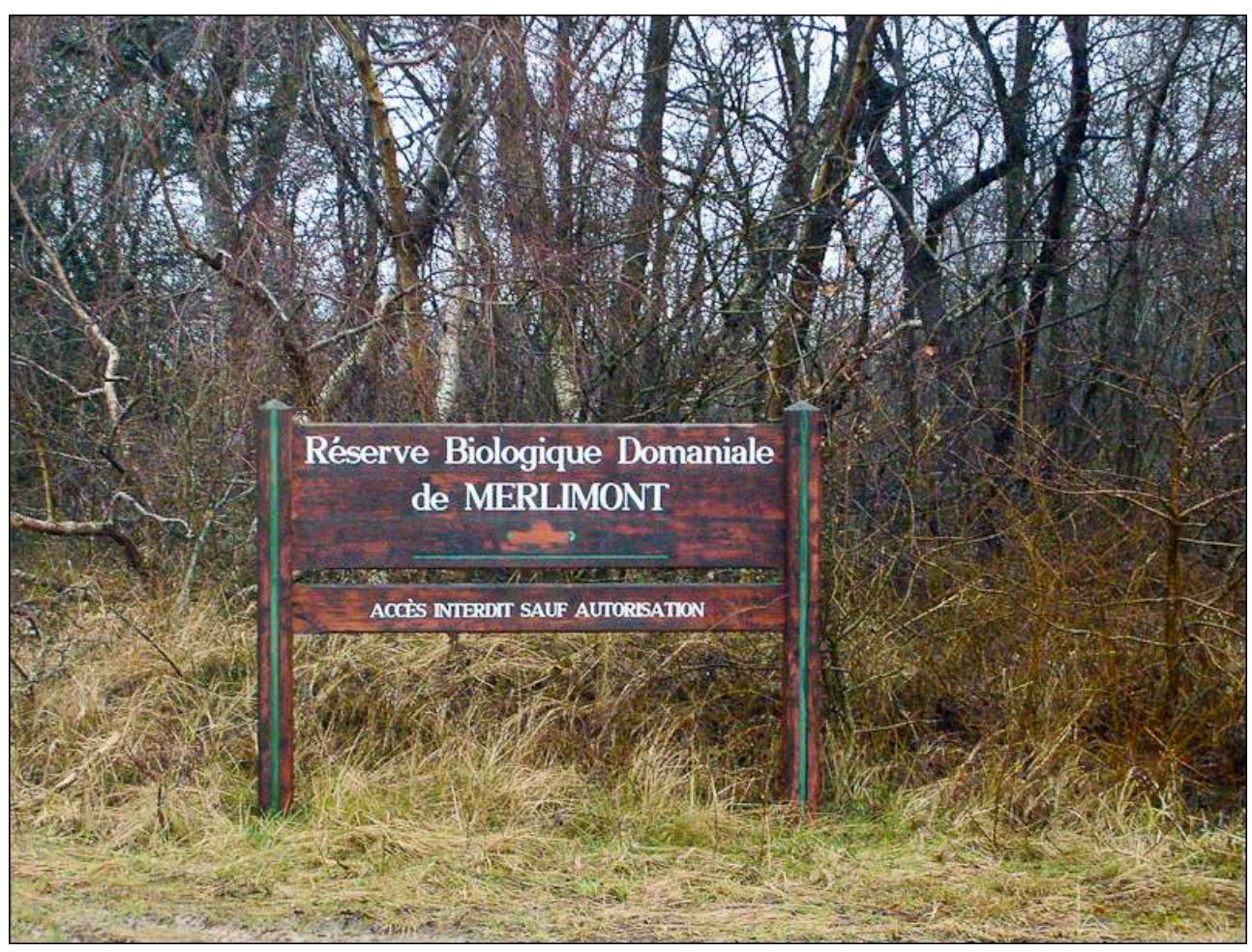

Source : cliché personnel de l'auteur, 2010.

\section{Choix de gestion d'hier et d'aujourd'hui}

10 La Réserve biologique domaniale est l'aboutissement d'un long processus d'appropriation et de valorisation d'un espace en proie à une dynamique permanente. 
Les facteurs écologiques tiennent une place prépondérante dans l'évolution du système dunaire: forte dynamique d'ensablement entretenue par de larges estrans et une abondance de sédiments, couvert végétal aux potentialités forestières mais fortement conditionné par les fluctuations des niveaux d'eau et l'action d'une faune lignivore agressive (lapins de garenne), topographie d'ensemble et de détail influençant la variété du modelé dunaire, l'existence de pannes et d'une large dépression pouvant servir de cadre à une activité cynégétique.

11 En dépit de l'importance de ces facteurs, il est clair que cet espace n'est jamais resté à l'écart des hommes et de leur volonté incessante de transformer un milieu qui leur paraissait aux premiers abords hostile. Dès le Moyen Age, l'Oyat, reconnu par ses qualités de rétention des particules de sable, est utilisé pour fixer le cordon dunaire externe. Comme pour d'autres dunes situées le long du littoral de la Manche, la plante n'est pas utilisée de manière assez rigoureuse, elle ne couvre pas toute l'étendue des dunes et est même arrachée par les villageois qui s'en servent pour se chauffer ou nourrir leur bétail. A la veille de la Révolution, dans le contexte difficile du Petit Age glaciaire, les dunes progressent sans relâche, ensevelissent les villages et les terres de culture. Dans un espace privé (garenne seigneuriale puis royale) mais fréquenté par la population locale (droit de parcours permettant une activité pastorale, chasse réglementée), les formations arbustives et arborées apparaissent alors peu développées sauf dans la large dépression où la colonisation du bouleau est déjà notée.

$\mathrm{Au} \mathrm{XIX}{ }^{e}$ siècle, deux évolutions sont notées : d'une part, une instabilité permanente des dunes vécue par les villageois et relatée dans les comptes-rendus des conseils municipaux, d'autre part, une orientation cynégétique du site avec l'aménagement de la plaine interdunaire au profit de quelques privilégiés avec création de mares, creusement de fossés, pour exercer la chasse au gibier d'eau. La dynamique du couvert végétal est alors contrôlée pour faciliter l'exercice de la chasse et les paysages évoluent peu. Le site forme ainsi une sorte d'enclave et tend à se dissocier des autres géosystèmes dunaires de la région marqués par une lente afforestation (boisement des dunes facilité par la mise en application du décret impérial du 14 décembre 1810) (Petit-Berghem, 2004). Il faut attendre la seconde moitié du XXe siècle pour assister à de réels et profonds changements. Plusieurs éléments jouent alors en faveur des arbres et de la constitution d'une forêt subnaturelle dominée par les feuillus. Tout d'abord, la chasse au lapin de garenne cumulée aux effets de la myxomatose favorise la dynamique forestière aux dépens des milieux ouverts, pelouses et prairies. Puis l'acquisition du site par l'État (fusion de quatre propriétés privées achetées entre 1972 et 1985) entraîne une valorisation sylvicole avec des tentatives d'imiter ce qui s'était fait en Aquitaine: fixation des cordons dunaires, plantations de pins (une quinzaine d'hectares de pin laricio entre 1975 et 1981), enrichissement par des feuillus (chênes, érables, trembles, etc.). L'objectif clairement affiché est alors de boiser le domaine, de l'aménager pour ensuite accueillir le public. Mais cette volonté ne reçut pas l'approbation de tous et suscita même de vives réactions dont celles des scientifiques et défenseurs de la nature opposés à ce projet. Il est vrai que des travaux émanant de personnalités reconnues pour leur qualité scientifique (Géhu et Wattez, 1978) avaient montré la nécessité de ne pas transformer à outrance un site trop rare à l'échelle européenne: diversité phytocénotique permettant l'expression d'un grand nombre de communautés végétales, forêt littorale relictuelle témoignant de la dynamique naturelle sur sable 
humide, richesse faunistique notamment aviaire servant de cadre à la reproduction d'oiseaux migrateurs, etc.

En 1992, dans une période favorable au renforcement de la protection de l'environnement, la Réserve biologique domaniale est choisie comme l'un des chantiers pilote du programme LIFE « Biodiversité et protection dunaire » encadré par la mission littorale de l'ONF. La réserve bénéficie à ce titre d'une série d'études renforçant les multiples intérêts de cet écocomplexe dunaire. La conservation des habitats trouve ainsi sa légitimité dans l'application des réglementations existantes (Code forestier, directives européennes de protection de la nature, arrêtés préfectoraux, Loi littoral, etc.). Il est choisi de faire de ce site l'un des référents en Europe pour mettre au point des méthodes de gestion adaptées à l'écologie et à la dynamique des milieux dunaires.

Les choix de gestion retenus pour la réserve découlent de cet objectif : favoriser la libre expression de la dynamique dunaire, maintenir ou restaurer des habitats à forte valeur patrimoniale, suivre et contrôler régulièrement l'évolution dynamique du site. Ces choix se justifient par la volonté de mieux comprendre le fonctionnement du système pour améliorer sa gestion. Mais ces choix naissent-ils d'une volonté commune ou du moins d'un consensus ? Reflètent-ils les demandes de la société ? Sont-ils en adéquation avec les élus qui sont aussi des financeurs? La réserve est-elle encore considérée comme une enclave ou participe-t-elle aux dynamiques territoriales?

\section{Les difficultés rencontrées}

Des difficultés sont liées aux relations partenariales en particulier lorsqu'il s'agit de s'entendre sur la régulation d'une pratique ancestrale très présente dans cet ensemble dunaire de plus de 800 ha. La chasse embarrasse l'ONF car elle entraîne des relations de nature commerciale telle que la location d'un droit par des acteurs pas toujours très sensibilisés aux questions environnementales. Aujourd'hui, l'accord entre préservation de la nature et pratique de la chasse repose sur la recherche d'un équilibre entre protection du milieu et exploitation de ses ressources. Cette pratique n'est pas facile à gérer car elle est enracinée dans le territoire et c'est sans doute elle qui a permis au site d'échapper à une urbanisation trop importante. En effet, les grands propriétaires terriens, tous chasseurs, ont précieusement préservé leur réserve de chasse de l'annexion foncière et par la suite immobilière (Almaric, 2003). Dès le XIX ${ }^{\mathrm{e}}$ siècle, les baux de chasse faisant l'objet d'adjudications se sont mis en place mais avec des structures, des dispositifs et des durées qui n'ont jamais été similaires d'une propriété à une autre. En 2010, dans le cadre du projet de classement des 290 ha propriété du Conservatoire du littoral en Réserve naturelle régionale mené avec la Région Nord-Pasde-Calais, les équipes du Conservatoire du littoral, du Syndicat mixte EDEN 62 gestionnaire du site, et de l'ONF ont entamé une réflexion d'harmonisation de la gestion à l'échelle du massif dunaire. Durant cette année, de nouveaux baux assortis d'une convention cynégétique ont été signés avec l'ONF, EDEN 62 et la Communauté de communes Opale Sud avec un zonage élargi à la rive nord de la baie d'Authie. Cet effort était essentiel pour garantir l'efficacité des actions de régulation menées par chacun sur l'ensemble du massif pour les espèces problématiques comme le sanglier. A cette fin, une gestion cynégétique coordonnée a été recherchée et mise en place entre et avec les différents acteurs locaux. 
16 D'autres tensions peuvent apparaître lorsqu'il s'agit de prendre des décisions sur les actions à mener tant dans leur mise en œuvre que dans le choix des zones à traiter. Certes, le plan d'action se base sur tout un arsenal de documents (cartographies des complexes d'habitats, inventaires, relevés périodiques, etc.) élaborés avec le plus grand soin, mais il ne permet pas d'éviter certains problèmes liés notamment aux prises de positions des scientifiques dont les propos n'entrent pas toujours en résonance avec les nécessités d'une gestion à court terme dont la priorité est d'assurer la sauvegarde d'espèces rares ou remarquables par le caractère exceptionnel de leur habitat ou de leurs caractéristiques locales. Ainsi, la démarche holistique prônée par la communauté scientifique est parfois difficile à appliquer car le temps de la réflexion est trop court (gestion à court terme limitant le coût des interventions) et les échelles spatiales d'application inadaptées (biotope voire écotope au détriment de l'ensemble du système dunaire). Afin de limiter les coûts, l'étendue des surfaces que le gestionnaire choisit de restaurer est directement dépendante de la surface qu'il lui sera financièrement possible d'entretenir régulièrement. Même au sein de la communauté scientifique, les intérêts divergent et les priorités ne sont pas toujours les mêmes bien qu'elles puissent toutefois se compléter. Un géomorphologue attiré par l'exceptionnelle diversité des formes dunaires sera favorable à la non intervention afin que les processus naturels puissent s'exprimer sans entrave et permettre ainsi l'observation de la dynamique dunaire. D'autres chercheurs sont au contraire favorables à des interventions visant à stopper ou ralentir des processus évolutifs (fixer un talus d'envahissement de sable, limiter des cuvettes de déflation, contrôler les écoulements de surface) notamment dans le cas où l'état de conservation d'habitats prioritaires n'est pas jugé satisfaisant. Les discussions au sein du comité consultatif de gestion permettent de peser le pour et le contre des propositions : les décisions reviennent au gestionnaire qui doit en amont intégrer les résultats des scientifiques quant à la compréhension du fonctionnement du milieu, et faire en aval avec les contraintes de chantier dont les techniques d'intervention sont en constante évolution.

17 Afin de réduire la facture de la restauration, l'ONF développe des collaborations avec des établissements scolaires préparant aux métiers forestiers ou d'environnement : les élèves réalisent des travaux pratiques in situ qui se résument bien souvent à l'apprentissage du maniement de la tronçonneuse ou de la débroussailleuse. Des sentiers nature sont également organisés par des associations et l'investissement de l'office est d'un coût minime puisqu'il s'agit la plupart du temps de bénévoles déjà sensibilisés à l'écologie de la restauration. Le coût d'entretien des zones restaurées est également l'objet de vifs débats: l'ONF a depuis quelques années compris qu'une coopération allant dans le sens d'une démarche de développement durable était nécessaire afin d'ancrer son action dans le temps et d'insérer la réserve dans une dynamique territoriale. Dès 2001, la rencontre avec un agriculteur local qui recherchait des prairies de fauche répondant au cahier des charges du label "Agriculture biologique ", permit d'engager une collaboration à bénéfice mutuel ( 7 à 8 hectares avec une somme de 150 euros versée par hectare fauché compte tenu de la qualité très moyenne du fourrage récolté). Afin de renouer avec les anciennes pratiques agricoles, l'ONF a également favorisé l'introduction d'un bétail rustique restant toute l'année sur place grâce à un complément de foin provenant de la réserve en hiver. Le coût d'intervention pour le gestionnaire se limita à la pose d'enclos alors que les frais liés à la constitution du troupeau (vaches de race "Highland Cattle»), son entretien et son suivi sanitaire étaient assurés par l'agriculteur. Cette méthode appuyée par le comité 
consultatif s'est avérée à la fois efficace (le suivi montre que le pâturage est l'outil qui semble donner le plus rapidement des résultats et avoir le plus d'effets sur la composition et la structuration de la végétation) et pertinente pour recréer un lien avec le passé et le territoire (pratiques agricoles à l'origine de nombreux milieux ouverts). Ce lien avec l'histoire se retrouve dans les micro-milieux liés à la Seconde guerre mondiale : sur un total de 240 trous de bombe recensés dans la réserve, 150 ont été réinvestis par une association environnementale («les Blongios») afin d'être convertis en mares offrant de nouvelles niches écologiques pour de nombreuses espèces d'amphibiens, d'odonates et d'hydrocantares (coléoptères aquatiques). Parallèlement, ces opérations spécifiques n'empêchent pas le gestionnaire d'agir plus globalement afin que les amphibiens puissent aussi bénéficier d'interventions initiées au profit d'autres communautés. C'est par exemple le cas des pannes dunaires où des travaux de débroussaillage sont localement entrepris pour conserver une plante remarquable (Epipactis des marais). L'objectif est alors de conserver la plus grande variété d'écosystèmes caractéristiques du littoral.

18 Si les opérations de restauration et de suivi écologique sont tout à fait concevables et même indispensables dans la vie d'une réserve, plusieurs projets dont certains ont abouti récemment montrent l'évolution de l'ONF en matière de stratégies de gestion et de communication. Depuis l'automne 2010, le public est par exemple autorisé à parcourir la réserve alors que certains habitants des stations balnéaires de Berck et de Merlimont ignoraient la présence d'un tel trésor à leur porte. Un sentier de randonnée sur pont de bois accessible à tous (y compris les personnes à mobilité réduite) s'offre aux visiteurs et permet de découvrir en surplomb toute la variété des écosystèmes dunaires (photographie 3). Axé sur la biodiversité du site et la fragilisation des milieux, le message est clair puisqu'il s'agit de sensibiliser le public en lui expliquant qu'une fréquentation excessive entraîne une détérioration ou une altération des milieux. On peut toutefois s'étonner que ce sentier ne ménage pas de réels points de vue d'ensemble permettant d'embrasser du regard toute la réserve et surtout la totalité du système dunaire. Cette perspective aurait permis aux visiteurs de découvrir tout le continuum littoral et les connexions entre unités paysagères. Il semble que l'accent ait été mis sur les différents milieux humides qui composent cet espace et qui en font pour les défenseurs de la nature un site hors du commun. Le message pédagogique très biocentrique laisse un peu perplexe : «Ici, on ne voit jamais la mer, mais on l'entend et on la sent " précise un représentant de l'ONF chargé du dossier (Queste, 2011). Cette vision dénote-t-elle un certain repli face à une ouverture quelque peu forcée ? Ou alors une stratégie marketing (ne pas trop en montrer mais bien dévoiler ce que fait l'office en matière de protection et de gestion conservatoire des milieux) nécessaire pour un site qui a pour vocation d'être une vitrine et un modèle de démonstration des processus de dynamique libre. En tout cas, elle reflète encore l'idéologie de l'ONF et va à l'encontre du schéma régional de la Trame verte et bleue du Nord-Pas-de-Calais qui considère les espaces littoraux comme une grande interface terre/mer (du milieu marin côtier à l'arrière côte) et un corridor naturel exceptionnellement riche, notamment au regard du rôle de ces milieux pour les migrations d'espèces (accueil des oiseaux hivernants, zones de haltes migratoires). Un autre sentier plus sportif et emmenant le public dans les arrières-dunes est stabilisé et recouvert d'écorces et offre des paysages plus végétalisés. Ces cheminements n'offrant pas de points de vue sur la mer qui est pourtant proche ont coûté près de 870000 euros, dont une aide de 500000 
euros du Conseil régional, le reste étant assumé par la Communauté de communes « Mer et Terres d'Opale ».

Photographie 3 - Le sentier de randonnée sur pont de bois

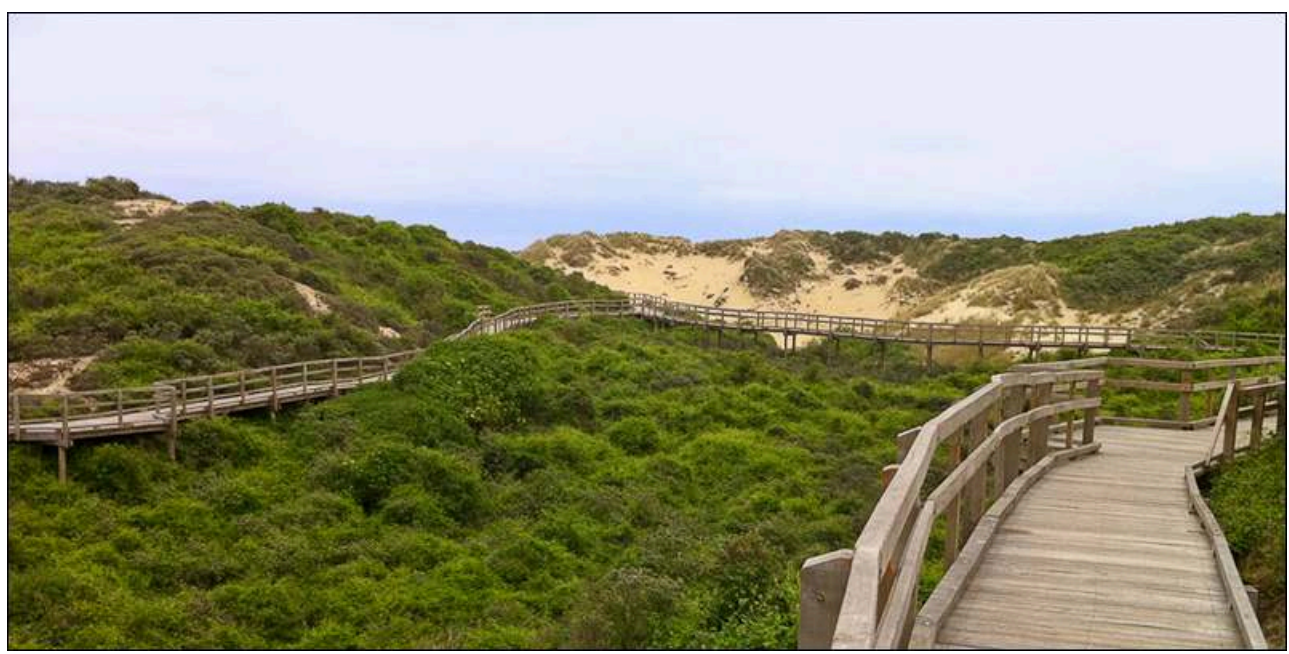

Source : cliché personnel de l'auteur, 2010 quelques années : il s'agit de la véloroute du littoral, piste cyclable touristique bordant les côtes européennes dont l'objectif est de pouvoir se déplacer en vélo à l'écart des grands axes. Ce projet inscrit dans le cadre de la Trame verte et bleue régionale est soutenu par l'Europe, le Conseil général, le Conseil régional, et la Communauté de communes. La réalisation de cette piste nécessite d'avoir une bonne liaison entre les communes : la pierre d'achoppement est le passage entre Merlimont et la piste cyclable de Berck où aucun itinéraire réalisable n'a encore émergé. C'est tout le paradoxe de l'hyperprotection explique Jean-Pierre Rapin, Maire de Merlimont et conseiller régional ( $83 \%$ du territoire de la commune est en zone protégée !). D’un côté un vaste marais (le marais de Balançon) protégé et privé à $98 \%$, de l'autre des dunes intégrées en grande partie dans la réserve biologique domaniale. Pourtant, un tracé à travers les dunes serait possible en utilisant la voie ferrée reliant autrefois Berck et Merlimont, et aujourd'hui ensablée. Mais l'ONF s'y refuse prétextant que les dunes bougent et ont un relief fragile. L'entretien d'une telle piste représente également un problème : qui se chargera de maintenir en état une infrastructure qui sera selon l'office sans cesse sous des centimètres de sable? Alors qu'une bonne liaison entre les communes représente un véritable atout pour le secteur, ce projet n'aboutit pas à l'inverse du précédent certes largement financé par la Région. On peut rester sceptique face à l'argumentaire de l'ONF qui n'a pas hésité à donner son consentement pour ouvrir simultanément deux sentiers pédestres susceptibles d'engendrer une fréquentation largement supérieure à la véloroute réservée aux cyclotouristes.

\section{Quel avenir ?}

Le succès et l'intérêt des réserves biologiques domaniales expliquent qu'elles ne soient plus cachées aujourd'hui du grand public (Merckelbagh, 2009). La réserve attire le scientifique qui désire expérimenter et comprendre le fonctionnement d'un milieu 
original, elle séduit l'usager ou le touriste itinérant friand de nature, de grands espaces, ou plus simplement d'un cadre agréable où il vient se ressourcer. L'avenir de la réserve dépend en grande partie de la politique impulsée par l'ONF: renforcement des partenariats existants, signature de nouveaux contrats ou de conventions avec les acteurs locaux, capacité à participer aux projets dépassant le cadre strict de la réserve, capacité d'auto-financement et subventions allouées par les collectivités et la sphère du privé, etc. Ouverture du public et conservation des écosystèmes seront deux tendances qui se poursuivront dans les années à venir : ces deux orientations qui étaient au départ presque dissociées (Meur-Férec, 2006) seront à coup sûr appelées à se rapprocher. Le discours de l'ONF évoluera-t-il ? Peut-être sera-t-il réajusté à la marge si la capacité d'ouverture de l'office s'élargit. Quoi qu'il en soit, il paraitt nécessaire de rappeler que la mentalité des forestiers a fortement évolué ces dernières années et que chaque agent est bien conscient que travailler en réseaux et avec de nombreux partenaires ne peut être qu'enrichissant et formateur. Mais, il faut également rappeler que la réglementation des réserves définit un cadre contraignant. Tout n'est pas permis et du temps est nécessaire pour s'apercevoir du bien-fondé d'une opération. Dans un espace complexe où la dimension historique prend une place prépondérante dans la dynamique du système, l'appréciation de l'efficacité des mesures de protection demande un recul important surtout en milieu forestier où les cycles sont particulièrement longs. Le plan de gestion révisé en 2006 reconduit les choix initiaux que dix ans d'expérience ont permis d'affiner.

21 L'objectif central reste de faire des dunes de Berck et de Merlimont un site pilote pour la connaissance et la gestion des milieux dunaires européens. Comme pour d'autres réserves en France ou à l'étranger, les prochaines années prolongeront la vocation «laboratoire grandeur nature " mis à la disposition des scientifiques: observer les dynamiques naturelles, s'appuyer sur ces dynamiques pour gérer la forêt et le milieu dunaire dans son ensemble. La réserve se doit de servir de cadre pour permettre une meilleure connaissance du milieu naturel. Mais comme le rappelle Jean Favennec, Chargé de mission «Littoral » à l'ONF et Chef de la mission littorale, ce site est aussi un espace de dialogue libre entre les porteurs des diverses attentes sociales.

\section{Conclusion}

La Réserve biologique domaniale de la Côte d'Opale relève du régime forestier et est gérée à ce titre par l'office national des forêts. Elle constitue avec celle de la Pointe d'Arcay en Vendée l'une des deux réserves côtières comportant des milieux dunaires et associés. Des connaissances ont été acquises depuis de nombreuses années tant au niveau des espèces et des habitats qu'au niveau des techniques de gestion. Il reste malgré tout de nombreuses questions sans réponse à l'heure actuelle. Se pose en particulier la valeur officielle du document de gestion en cas de conflit d'usage. Ce document établi dans le jeu de la concertation ne reflète pas les souhaits de tous les acteurs. Quoi qu'il en soit, il importe de poursuivre les efforts en faveur d'une gestion globale expérimentale et de suivre l'évolution de cette réserve, dans son propre fonctionnement et dans ses rapports avec le milieu riverain.

23 L'homme a pratiqué sur ce site diverses activités qui ont marqué le paysage. L'anthropisation très ancienne du domaine plaide en faveur d'un renforcement de son ouverture au public: l'accès y est favorisé depuis moins d'un an et cet ensemble 
dunaire ne constitue plus un espace réservé aux seuls scientifiques. Le grand public découvre grandeur nature cette réserve mais sa perception est un peu biaisée en raison du parti pris de l'ONF et de sa volonté de garder la main sur le discours scientifique. La notoriété de l'établissement en dépend. Gageons que le renforcement des liens entre usagers, acteurs territoriaux, et forestiers permettra par les échanges et les réflexions d'assurer la pérennité d'un système dunaire exemplaire dont la reconnaissance par une meilleure connaissance s'en trouvera elle-même bonifiée.

\section{BIBLIOGRAPHIE}

Almaric M. (coord.), 2003. Perception et aménagement des zones humides : l'apport de l'étude des représentations dans la gestion d'une zone naturelle menacée. L'exemple du site de Berck-Merlimont. Université des Sciences et Technologies de Lille, $40 \mathrm{p}$.

Dereix Ch. (coord.), 2001. Dans les dunes de Merlimont : la réserve biologique de la Côte d'Opale. Arborescences, 91, p. 2-27.

Dermaux B., 2007. Réserve biologique domaniale de la Côte d'Opale, choix de gestion hier et aujourd'hui. RDV techniques de l'ONF, $\mathrm{n}^{\circ} 17, \mathrm{p} .40-43$.

Favennec J., 2007. Les dunes de Merlimont-Côte d'Opale, site d'excellence pour la connaissance et la gestion des milieux dunaires européens, $R D V$ techniques de l'ONF, $\mathrm{n}^{\circ} 17, \mathrm{p}$. 31-34.

Favennec J. (coord.), Barrère P. (dir. scientifique), 1997. Biodiversité et protection dunaire. Paris, Technique et Documentation Lavoisier, $311 \mathrm{p}$.

Géhu J.-M., Wattez J.-R., 1978. La forêt littorale des dunes de Merlimont (Ligustro-Betuletum pubescentis). Documents phytosociologiques, N.S., vol II, p. 195-203.

Merckelbagh A., 2009. Et si le littoral allait jusqu'à la mer ! La politique du littoral sous la Ve République. Paris, Quæ, $351 \mathrm{p}$.

Meur-Férec C., 2006. De la dynamique naturelle à la gestion intégrée de l'espace littoral : un itinéraire de géographe. Habilitation à diriger des recherches, Université de Nantes, vol I, 247 p.

Paskoff R., 2003. La conservation des dunes littorales implique-t-elle leur stabilisation ? L'exemple de la côte atlantique. Natures Sciences Sociétés, 11, p. 288-294.

Petit-Berghem Y., Dubois J.-J., 1995. Les forêts littorales en milieu dunaire : l'exemple de la réserve biologique domaniale de Merlimont (Pas-de-Calais). Hommes et Terres du Nord, 1-2, p. 11-20.

Petit-Berghem Y., 2004. Formes de perception et de gestion des forêts dunaires françaises depuis leur création. In Taabni M. (dir.), La forêt : enjeux comparés des formes d'appropriation, de gestion et d'exploitation dans les politiques environnementales et le contexte d'urbanisation généralisé, Poitiers, Maison des Sciences de l'Homme et de la Société, p. 273-282.

Queste B., 2011. Idée de promenade à Merlimont, le sentier de découverte de la réserve biologique dunaire, http://www.echo62.com, consulté le 27/07/11. 


\section{NOTES}

1. Petite dépression dunaire soumise aux battements saisonniers de la nappe phréatique.

\section{RÉSUMÉS}

La Réserve biologique domaniale de la Côte d'Opale représente en France l'un des rares systèmes dunaires marqués par la libre expression des processus dynamiques. La mobilité du site garantit le renouvellement et la diversité des biotopes et donc des habitats et des espèces qui leur sont associées. Comme toute réserve, il s'agit d'un espace réglementé où les actions de gestion sont orientées dans un sens conservatoire et strictement contrôlées. La difficulté des gestionnaires est aujourd'hui de définir un objectif à plus long terme de cette réserve. Quelles sont les structures partenaires à privilégier? Quel degré d'ouverture au public? Quelles sont les pistes de recherche à développer? Comment concilier une politique de conservation écologique avec un développement durable du territoire? Ces différents questionnements appellent une réflexion entre nature et société et renvoient à la gouvernance des territoires et à la place de l'homme dans son environnement.

The coastal dunes of the Pas-de-Calais area (dunes of Berck-Merlimont with the "Réserve biologique domaniale de la Côte d'Opale ", northwest France) is one of the few dune systems in France characterized by the free expression of dynamic processes. The mobility of the site ensures the renewal and diversity of dune environments and thus habitats and species associated with them. Like any reserve, it is a regulated area where management actions are oriented in one direction (conservation management) and strictly controlled. The challenge for managers today is to define a longer-term objective of this reserve. What are the working partners for which it would be possible to collaborate? What degree of openness to the public? What are the research topics to develop? How to reconcile a policy of environmental conservation with sustainable territorial development? These questions require a discussion between nature and society and return to the governance of the territories and the place of man in his environment.

\section{INDEX}

Mots-clés : réserve biologique, dune, biodiversité, gestion, développement durable Keywords : biological reserve, dune, biodiversity, management, sustainable development Thèmes : Sur le Champ - Sur le Terrain

\section{AUTEUR}

\section{YVES PETIT-BERGHEM}

Yves Petit-Berghem yves est maître de conférences à l' Université de Caen Basse-Normandie et membre de LETG - UMR 6554 CNRS, Laboratoire Géophen. yves.petit-berghem@unicaen.fr 